\title{
Discovery and Fine Mapping of qSCR6.01, a Novel Major QTL Conferring Southern Rust Resistance in Maize
}

\author{
Lu Lu, ${ }^{1}$ Zhennan Xu, ${ }^{1}$ Suli Sun, ${ }^{1}$ Qing Du, ${ }^{2}$ Zhendong Zhu, ${ }^{1}$ Jianfeng Weng, ${ }^{1, \dagger}$ and Canxing Duan ${ }^{1, \dagger}$ \\ ${ }^{1}$ Institute of Crop Sciences, Chinese Academy of Agricultural Sciences, National Key Facility for Crop Gene Resources and Genetic \\ Improvement, Beijing 100081, China \\ ${ }^{2}$ Institute of Maize Research, Guangxi Academy of Agricultural Sciences, Nanning 530007, China
}

\begin{abstract}
Southern corn rust (SCR), an airborne disease caused by Puccinia polysora, can severely reduce the yield of maize (Zea mays L.). Using recombinant inbred lines (RILs) derived from a cross between susceptible inbred line Ye478 and resistant Qi319 in combination with their highdensity genetic map, we located five quantitative trait loci (QTLs) against SCR, designated as qSCR3.04, qSCR5.07, qSCR6.01, qSCR9.03, and $q S C R 10.01$, on chromosomes 3, 5, 6, 9, and 10, respectively. Each QTL could explain 2.84 to $24.15 \%$ of the total phenotypic variation. qSCR6.01, detected on chromosome 6, with the highest effect value, accounting for $17.99,23.47$, and $24.15 \%$ of total phenotypic variation in two environments and best linear unbiased prediction, was a stably major

resistance to SCR. The line CL183 harboring introgressed qSCR6.01 showed obvious resistance to SCR that was distinctly different from that of Ye478 $(P=0.0038)$. Further mapping of $q S C R 6.01$ revealed that the resistance QTL was linked to insertion-deletion markers Y6q77 and Y6q79, with physical locations of 77.6 and $79.6 \mathrm{Mb}$, respectively, on chromosome 6. Different from previous major genes or QTLs against SCR on chromosome 10, qSCR6.01 was a newly identified major QTL resistance to SCR on chromosome 6 for the first time. Using RIL and CSSL populations in combination, the SCR-resistance QTL research can be dissected effectively, which provided important gene resource and genetic information for breeding resistant varieties.
\end{abstract} resistance QTL. The common confidence interval for $q S C R 6.01$ was 2.95 $\mathrm{Mb}$ based on the B73 RefGen_v3 sequence. The chromosome segment substitution lines (CSSLs) constructed with Qi319 as the donor parent and Ye478 as the recurrent parent were used to further verify $q$ SCR6.01
Keywords: chromosome segment substitution lines, cultivar/resistance, disease management, field crops, maize, resistance, resistance gene, QTL mapping, recombinant inbred lines, southern corn rust
Maize (Zea mays L.) is a major crop, and its yields affect national food security in China. Outbreaks of southern corn rust (SCR), a leaf disease caused by Puccinia polysora Underw., remarkably reduce maize production. After pathogen infection, the typical symptom of SCR is the appearance of bright orange-red, 1- to 2-mm, round and oval pustules. SCR was first discovered in Africa, where it was identified as a tropical maize disease (Cammack 1954; Rhind et al. 1952).

In China, SCR was first detected in Hainan Province in 1972 (Duan and He 1984). As a result of climate change, cultivar alternation, and susceptible varieties deploying and more pathogen coming, SCR has become the main disease in maize-producing fields in southern China, such as in Hainan, Guangdong, Guangxi, Jiangsu, and Zhejiang Provinces, where yield losses as high as 50\% were recorded (Liu et al. 1999; Wang 1999). In recent years, SCR has also posed a serious threat to maize production in the Huang-Huai-Hai region (Liu et al. 2009).

The most economical and effective method of damage control against SCR is breeding and cultivating resistant varieties. Screening

${ }^{\dagger}$ Corresponding authors: C. Duan; duancanxing@ caas.cn; and J. Weng; wengjianfeng@ caas.cn

Funding: This project was supported by the National Key Research and Development Program of China (2016YFD0100103), Special Fund for Protection of Crop Germplasm Resources (2019NWB036-12), and the Agricultural Science and Technology Innovation Program of the Chinese Academy of Agricultural Sciences.

*The $\boldsymbol{e}$-Xtra logo stands for "electronic extra" and indicates that three supplementary figures and three supplementary tables are published online.

The author(s) declare no conflict of interest.

Accepted for publication 12 February 2020

(C) 2020 The American Phytopathological Society resistant resources, and mining and mapping resistance genes or quantitative trait loci (QTLs), are the bases for resistance breeding. Since the 1950s, some resistance genes against SCR have been excavated and mapped. The resistance genes Rppl and Rpp2 were first identified, conferring specific resistance to $P$. polysora races EA1 and EA2, respectively (Storey and Howland 1959). The dominant gene $R p p 9$, expressing resistance to race pp.9, was located on the short arm of chromosome 10 (Ullstrup 1965). Another resistance gene showing specific resistance to race pp.9 was identified by Futrell et al. (1975). Using simple sequence repeat (SSR), cleavedamplified polymorphic sequence, and insertion-deletion (InDel) polymorphic markers, the dominant resistance genes $R p p P 25, \operatorname{Rpp} Q$, $R p p D, R p p C$, and $R p p S$ were mapped on the short arm of chromosome 10 (Chen et al. 2004; Liu et al. 2003; Wu et al. 2015; Yao et al. 2013; Zhang et al. 2010; Zhou et al. 2007). In addition to major genes, some minor QTLs associated with SCR resistance have been mapped as well. Up to now, QTLs with partial resistance to SCR have been detected on all maize chromosomes (Brewbaker et al. 2011; Brunelli et al. 2002; Chen et al. 2019; Deng et al. 2019; Holland et al. 1998; Jines et al. 2007; Wanlayaporn et al. 2013; Wisser et al. 2006). These studies will provide valuable information for resistance breeding.

Linkage mapping is an important method for studying heredity and variation of complex agronomic or resistant genes. Molecular markers such as SSR and single-nucleotide polymorphism (SNP) were used to construct genetic linkage maps. However, many previous studies used the limited number of markers for QTL mapping, which limits the efficiency and accuracy of QTL localization and reduces the coverage of genetic markers (Liu et al. 2011; Sala et al. 2006; Z. H. Wang et al. 2012; Z. Q. Wang et al. 2012). Genotypingby-sequencing (GBS) using high-throughput sequencing allows the large-scale development of genetic markers for the construction of high-density genetic maps over a short period of time (Elshire et al. 2011). The construction of an ultrahigh-density genetic map based on high-quality recombinant inbred lines (RILs), an effective method for the QTL localization of complex traits, can be applied for the detection and mapping of QTLs (Zhang et al. 2017; Zuo et al. 2015). 
In maize, RIL populations have been widely used to identify and map resistant genes and QTLs (Ding et al. 2008; Wang et al. 2018). However, as a primary mapping population, RILs have a complex genetic background and are susceptible to environmental interference (Alonso-Blanco and Koornneef 2000; Eshed and Zamir 1994). Chromosome segment substitution lines (CSSL) can reduce interference from the genetic background and have been proved to be suitable for mapping, confirmation, and cloning of target genes and QTLs. Among CSSL, most segments throughout the genome have the same background, with only one or a few different fragments. Therefore, the CSSL approach can be used to eliminate the interference of other backgrounds and achieve QTL fine mapping. CSSLs have been widely used for the localization and cloning of genes and QTLs to control important agronomic traits in maize (Bangtai et al. 2012; Li et al. 2014; Lopez-Zuniga et al. 2019; Zhang et al. 2011).

In the present study, a superior maize inbred line, Qi319, was selected as the disease-resistant parent, and Ye478 was chosen as the susceptible parent, for generation of an RIL population. Using this population in combination with an extremely high-density linkage map constructed from 4,183 pairs of bin markers uniformly distributed across the entire chromosomes 1 to 10 , we located five QTLs for SCR resistance. In addition, we developed relevant CSSLs to carry out the fine mapping and evaluation of QTLs for SCR resistance to confirm their potential and refine previously mapped regions. Because the genetic diversity of maize lines with resistance to SCR is relatively poor (Chen et al. 2018; Duan et al. 2015), the identification of QTLs for SCR resistance is of great importance for maize disease control and germplasm improvement.

\section{Materials and Methods}

Plant materials. Inbred line Qi319 was developed from maize 78599, a hybrid of non-Chinese origin selected by the Shandong Academy of Agricultural Sciences that is highly resistant to SCR (Tian et al. 2014). Ye478 is an inbred line with a maize backbone having the advantages of excellent comprehensive agronomic traits and high general combining ability (Zheng et al. 2009). A RIL population composed of $300 \mathrm{~F}_{11}$ lines was constructed by the single-seed descent method using Qi319 and Ye478 as parents. CSSLs were bred using Qi319 and Ye478 as donor and receptor parents, respectively. After back-crossing for five generations and self-crossing for three, six CSSLs were selected to verify the SCR-resistance QTL on chromosome 6. The six CSSLs were CL184, CL183, CL93, CL95, CL98, and CL99, designated as type1 to type6. Type2 (CL183) is the CSSL with resistant gene. The $\mathrm{F}_{2}$ hybrid generation derived from CL183 and Ye478 was used for subsequent gene mapping.

Phenotypic evaluation of maize for SCR resistance. In September 2017, RILs, Qi319, and Ye478 were planted in Nanning, where the temperature and moisture are suitable to facilitate pathogen $P$. polysora invasion to cause disease from October to December. In 2018, these materials were planted at a different site in Nanning in September and inoculation was carried out in October. The field experiment was based on a randomized block design with two replicates. The plot consisted of 600 rows, $5 \mathrm{~m}$ long per row, row spacing of $0.6 \mathrm{~m}$, and plant spacing of $0.25 \mathrm{~m}$. There were 17 to 20 plants in each row. Control groups (Qi319 and Ye478) were set out every 50 rows. The climatic conditions are suitable for the occurrence of SCR in spring in Hainan Province. In January 2019, six CSSLs covering chromosome 6 and five $F_{2}$ hybrid generations derived from CL183 and Ye478 were planted in Sanya, Hainan. Inoculation was carried out in February 2019, and a series of surveys for SCR on the field-grown CSSLs was conducted in March 2019. The SCR strain for inoculation, PPGN, was collected from Nanning, Guangxi Province, then multiplied on susceptible maize inbred line Huangzaosi in a greenhouse. To prepare a suspension for inoculation, the Huangzaosi maize leaves covered with $P$. polysora uredospores were manually washed and filtered. About 7 to $8 \mathrm{ml}$ of uredospore suspension with a concentration of $6 \times 10^{4}$ spores $/ \mathrm{ml}$ was sprayed onto each maize plant with sprayers at the eight- to nine-leaf stage. The first survey was carried out before the tasseling stage, with a second survey performed at the grain-filling stage. All materials were investigated in RIL and CSSL populations, and the disease scale, which was based on the disease symptom descriptions of Wang (2005), was recorded. Disease resistance was evaluated according to the disease grade, as shown in Supplementary Table S1 (Wang et al. 2010).

Analysis of phenotypic data and heritability. All descriptive statistics (mean, range, skewness, and kurtosis) of the parental lines and RILs across the two environments were analyzed using Statistical Product and Service Solutions (SPSS) software v20.0. The generalized heritability $\left(\mathrm{H}^{2}\right)$ of SCR resistance in the two environments was analyzed using the method of Wang (2017). Standard analysis of variance with the general linear model procedure (PROC GLM) was adopted to estimate all variance in SAS 9.0. We used the genetic model $H^{2}=\sigma_{\mathrm{G}}{ }^{2} /$ $\left(\sigma_{\mathrm{G}}^{2}+\sigma_{\mathrm{G} \varepsilon}{ }^{2} / \mathrm{e}+\sigma_{\varepsilon}{ }^{2} / e r\right)$, where $\sigma_{\mathrm{G}}{ }^{2}$ is the genetic variance that reflects blocking effect, $\sigma_{\varepsilon}^{2}$ is the error variance, $\sigma_{\mathrm{G} \varepsilon}{ }^{2}$ is the genotypeenvironment interaction, and $e$ and $r$ are the number of environments and repeats of an environment, respectively. Inclusive composite interval mapping (ICIM) was used to locate QTLs (Wang 2009).

Genetic linkage map construction and QTL mapping analysis for SCR resistance in the RIL population. The GBS approach on an Illumina HiSeq2500 system was used to genotype the 300 RILs and their parents. B73 RefGen_V3 was selected as the reference genome. After filtering raw reads generated by sequencing, 137,699,000 reads were obtained, with an average of 357,376 reads per individual. In total, 88,268 SNPs were developed, of which 4,183 polymorphic SNPs between Qi319 and Ye478 were finally selected and used to construct the linkage map. A high-density genetic map covering the 10 chromosomes with a total length of 1,545.65 centimorgans (cM) was constructed. The average genetic distance between markers was $0.37 \mathrm{cM}$, with a physical distance of $0.51 \mathrm{Mb}$ (Zhou et al. 2016).

ICIM was used for QTL detection in IciMapping v4.0 software (Meng et al. 2015; Wang 2017). The method of ICIM can exclude the influence of other QTLs outside the current interval. Best linear unbiased prediction (BLUP) was calculated with the model BLUP = $\left(\mu_{1}+\mu_{2}\right) / e$, where $\mu_{1}$ and $\mu_{2}$ are the average performance in E1 (2017 in Nanning, Guangxi Province) and E2 (2018 in Nanning, Guangxi Province) and $e$ is the number of environments (Wang 2017). The threshold of logarithm of odds (LOD) scores for evaluating the QTL effects at a significant level of $P=0.05$ were determined using 1,000 permutations (Doerge and Churchill 1996; Li et al. 2007). The previous studies identified LOD thresholds ranging from 2.97 to 3.56, with a mean value of 3.0 as a conservative cutoff (Zhou et al. 2016). Therefore, a LOD threshold of 3.0 was used to claim the presence of putative QTL.

Validation and mapping the qSCR6.01 loci using a CSSL population. After generating a CSSL population with elite inbred line Qi319 as the donor and Ye478 as the receptor, we performed marker-assisted selection (MAS) based on 201 polymorphic SSR markers, with an average interval of $9.94 \mathrm{Mb}$ between markers, uniformly distributed on the chromosome to obtain 200 CSSLs covering the complete maize chromosome.

The genetic background of the CSSLs was evaluated by computing the percentage of the recurrent genome based on molecular marker techniques. The formula used for the calculation was $G_{g}=$ $1 / 2+(1 / 2)\left(X_{g} / L\right)$, where $G_{g}$ is the percentage of the recurrent genome in generation $g, X_{g}$ is the number of molecular-marker amplification bands obtained in the CSSL in backcross-generation $g$ that were also found in the recurrent parent, and $L$ is the total number of molecular markers (Hospital et al. 1996). The length of substituted segments was calculated by the method of Young and Tanksley (1989).

Ignoring the possibility of double-crossover events between two markers, we used the following procedure to identify donor segments. When the genotypes of two adjacent markers were consistent with that of the genotype of Qi319 (donor), the segment between the two markers was considered to be the substituted segment from the donor parent. New markers were added until the genotype of the nearest adjacent marker was consistent with that of Ye478 (receptor); that midpoint between the outermost marker of the substituted segment and the adjacent receptor marker was then identified as the critical site of the substituted segment. The length between two critical sites is the length of substituted segment (Wang et al. 2018; Young and Tanksley 1989). 
To finely map the SCR-resistance gene on chromosome 6, InDel markers (Supplementary Table S2) were designed to increase the marker density between 70 and 80 Mb (B73 RefGen_v3). PCR amplifications were performed on a GeneAmp PCR System 9700 thermal cycler (ABI, Norwalk, CT, U.S.A.). The PCR amplifications were performed in $20-\mu \mathrm{l}$ volumes containing $50 \mathrm{ng}$ of genomic DNA, $0.2 \mu \mathrm{M}$ primer mixture, $1.5 \mathrm{U}$ of Taq DNA polymerase, $0.2 \mathrm{mM}$ dNTPs, and $2.0 \mu \mathrm{l}$ of $10 \times$ buffer. The cycling protocol was as follows: $94^{\circ} \mathrm{C}$ for $4 \mathrm{~min}$; followed by 35 cycles of $94^{\circ} \mathrm{C}$ for $1 \mathrm{~min}, 50$ to $60^{\circ} \mathrm{C}$ for $1 \mathrm{~min}$, and $72^{\circ} \mathrm{C}$ for $1 \mathrm{~min}$; with a final extension at $72^{\circ} \mathrm{C}$ for $10 \mathrm{~min}$.

\section{Results}

Phenotypic variation in resistance to SCR in RILs. Phenotypic observations of 300 RILs and evaluation for resistance to SCR were carried out in the field in 2017 and 2018 in Nanning, Guangxi. The observed phenotypic variation is detailed in Table 1 .

Significant differences in SCR resistance $\left(P=3.08 \times 10^{-10}\right)$ were identified between Qi319 and Ye478 from the phenotypic survey (Supplementary Fig. S1). Transgressive segregation of SCR resistance was observed in the RILs, and a large variation in resistance was found among different families. The scales of SCR resistance among RILs, which ranged from completely susceptible to highly resistant, were close to normally distributed (Supplementary Fig. S2), which indicates that SCR resistance is a quantitatively inherited trait that can be analyzed by QTL mapping. According to estimates based on analysis of the contributions of environmental and genetic determinants to SCR resistance, $H^{2}$ was as high as $91.98 \%$, thus indicating that the phenotypic variance in the RIL population was predominantly controlled by genetic factors (Table 2).

QTL mapping of SCR resistance in the RIL population. In 2017, four QTLs, tentatively designated as qSCR3.04, qSCR6.01, $q S C R 9.03$, and $q S C R 10.01$, located on chromosomes 3, 6, 9, and 10 , respectively, were detected in Nanning, with corresponding LOD values of $3.18,19.86,3.74$, and 24.33 , respectively, that explained 2.29 to $21.21 \%$ of the total variation in the phenotype (Table 3). The contribution rate of the QTLs on chromosomes 6 and 10 was more than $10 \%$; namely, 17.99 and $21.21 \%$, respectively. Three QTLs, located on chromosomes 3, 6, and 10, were detected in the second environment (in 2018, Nanning) and could explain 2.79 to $23.47 \%$ of the phenotypic variation. qSCR6.01 on chromosome 6 associated with SCR resistance had the maximum phenotypic variation (23.47\%). qSCR3.04, qSCR6.01, and qSCR10.01, all donated by Qi319, were stably detected in both environments; among these three resistance alleles, $q S C R 6.01$ and $q S C R 10.01$ had high additive effects and LOD values in both environments. Using a LOD threshold of 3.0 (Fig. 1) and a 95\% confidence interval, five QTLs, on average, were detected on chromosomes 3, 5, 6, 9, and 10. qSCR6.01 and $q S C R 10.01$ had large LOD values (27.98 and 26.42, respectively) and their additive effects were -2.36 and -2.32 . The negative additive effect showed that the allele carried by Qi319 had the effect of heightening SCR resistance. qSCR6.01, which explained $24.15 \%$

Table 1. Phenotypes of parental lines and a recombinant inbred lines (RIL) population based on two replicates

\begin{tabular}{|c|c|c|c|c|c|c|c|c|}
\hline \multirow[b]{2}{*}{ Year ${ }^{\mathrm{a}}$} & \multirow{2}{*}{$\frac{\text { Qi319 }}{\text { Mean }}$} & \multirow{2}{*}{$\frac{\text { Ye478 }}{\text { Mean }}$} & \multicolumn{6}{|c|}{ RILs $^{\mathbf{b}}$} \\
\hline & & & Mean \pm SD & Range & Skewness & Kurtosis & $\mathrm{CV}(\%)$ & $H^{2}(\%)$ \\
\hline E1 & 3.6 & 7.0 & $5.40 \pm 2.00$ & $3.0-7.0$ & -0.538 & -1.449 & 4.013 & \\
\hline E2 & 3.3 & 9.0 & $5.52 \pm 1.93$ & $3.0-7.0$ & -0.549 & -1.706 & 3.72 & 91.98 \\
\hline Combined & 3.4 & 8.0 & $5.46 \pm 1.97$ & $3.0-7.0$ & -0.545 & -1.556 & 3.865 & \\
\hline
\end{tabular}

a E1 and E2 indicate Nanning, Guangxi Province, in 2017 and 2018, respectively.

b $\mathrm{SD}=$ standard deviation, $\mathrm{CV}=$ coefficient of variation, and $H^{2}=$ generalized heritability.

Table 2. Analysis of variance of southern corn rust resistance ${ }^{\mathrm{a}}$

\begin{tabular}{lccccc}
\hline Source & df & SS & MS & $\boldsymbol{F}$ value & $\boldsymbol{P r}>\boldsymbol{F}$ \\
\hline Genotype (G) & 299 & $1,928.0367$ & 6.4482832 & 1.7195422 & $1.358 \mathrm{E}-08$ \\
Environment (E) & 1 & 0.0033333 & 0.0033333 & 0.0008889 & 0.9762251 \\
G $\times$ E & 299 & 266.99667 & 0.8929654 & 0.2381241 & 1 \\
Residuals error & 600 & 2,250 & 3.75 & $\ldots$ & $\ldots$ \\
\hline
\end{tabular}

${ }^{a} \mathrm{df}=$ degree of freedom, $\mathrm{SS}=$ sum of squares, MS = mean square deviation, and $H^{2}=$ generalized heritability.

Table 3. Quantitative trait loci (QTL) mapping for southern corn rust resistance using a recombinant inbred line population derived from Qi319 and Ye478 ${ }^{\text {a }}$

\begin{tabular}{|c|c|c|c|c|c|c|c|}
\hline $\mathrm{Chr}^{\mathbf{b}}$ & Peak position & Left marker & Right marker & Interval (B73_v3, Mb) & LOD & PVE (\%) & ADD \\
\hline \multicolumn{8}{|l|}{ E1 } \\
\hline 3 & 59.5 & mk1350 & mk1356 & $56.25-68.70$ & 3.18 & 2.29 & -0.87 \\
\hline 6 & 77.7 & $\mathrm{mk} 2873$ & $\mathrm{mk} 2844$ & $74.65-78.55$ & 19.86 & 17.99 & -2.37 \\
\hline 9 & 100.15 & mk4143 & mk4159 & $97.25-102.80$ & 3.74 & 2.43 & 0.85 \\
\hline 10 & 4.05 & mk4329 & mk4336 & $3.15-4.55$ & 24.33 & 21.21 & -2.54 \\
\hline \multicolumn{8}{|l|}{ E2 } \\
\hline 3 & 68.7 & mk1355 & mk1357 & $59.50-77.50$ & 3.15 & 2.79 & -0.8 \\
\hline 6 & 73.3 & mk2834 & mk2839 & $69.90-75.85$ & 23.6 & 23.47 & -2.27 \\
\hline 10 & 3.65 & $\mathrm{mk} 4328$ & $\mathrm{mk} 4336$ & $3.05-4.55$ & 17.01 & 15.25 & -1.81 \\
\hline \multicolumn{8}{|l|}{ BLUP } \\
\hline 3 & 68.7 & mk1355 & mk1357 & $59.50-77.50$ & 4.24 & 3.03 & -0.86 \\
\hline 5 & 207.4 & mk2704 & $\mathrm{mk} 2725$ & $204.80-210.05$ & 3.05 & 3.11 & 0.73 \\
\hline 6 & 78 & $\mathrm{mk} 2838$ & $\mathrm{mk} 2843$ & $75.30-78.25$ & 27.93 & 24.15 & -2.36 \\
\hline 9 & 99.45 & $\mathrm{mk} 4142$ & $\mathrm{mk} 4155$ & $96.80-101.60$ & 3.91 & 2.84 & 0.8 \\
\hline 10 & 4.05 & mk4329 & $\mathrm{mk} 4355$ & $3.15-4.45$ & 26.42 & 22.98 & -2.32 \\
\hline
\end{tabular}

a Peak position = position with the highest logarithm of odds (LOD), Left marker = marker to the left of the QTL, Right marker = marker to the right of the QTL, Interval = interval between the two markers on the B73 RefGen_v3 genome sequence, PVE = phenotypic variance of a single QTL, and ADD = additive effects of one QTL.

b Environment and chromosome (Chr): E1 and E2 = 2017 and 2018, respectively, in Nanning, Guangxi Province, and BLUP = best linear unbiased prediction. 
of the observed phenotypic variation, was flanked by markers mk2838 to mk2843, with physical distances of 75.30 to $78.25 \mathrm{Mb}$ (B73 RefGen_v3).

Validation and mapping the qSCR6.01 loci using a CSSL population. The effect of $q S C R 6.01$ was investigated in a CSSL population derived from a cross between Qi319 and Ye478 that carried different types for the segments covering chromosome 6. The six CSSLs were obtained using the MAS method $\left(\mathrm{BC}_{5} \mathrm{~F}_{3}\right)$ by backcrossing five generations and three generations. These six CSSLs with the average background recovery rates by molecular MAS $\left(\mathrm{BC}_{5} \mathrm{~F}_{3}\right)$ ranged from 91.45 to $99.62 \%$. Drawing on the results of QTL mapping of the RIL population, six CSSLs covering chromosome 6 were constructed to map qSCR6.01. The results of phenotypic analysis of the CSSLs are shown in Figure 2. CL183 and CL184 exhibited resistance to SCR, whereas the other CSSLs covering chromosome 6 were susceptible (Supplementary Fig. S3; Supplementary Table S3). According to the field phenotypes, CL183, with a resistance rating of 4.5 , was

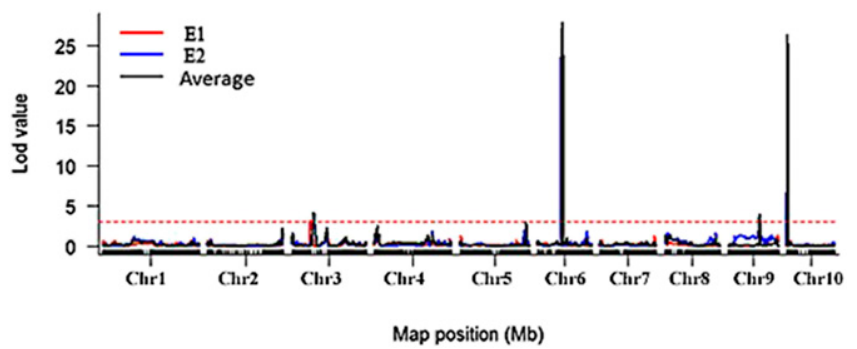

Fig. 1. Detection of southern corn rust (SCR) resistance quantitative trait loci (QTLs) in two environments. The diagram of QTLS for SCR resistance throughout the entire genome is based on 300 recombinant inbred lines derived from the cross Ye478 $\times$ Qi319. The display of the logarithm of odds (LOD) profile, relative positions, and relevant markers was created using QTL cartographer v3.5. E1 and E2 refer to 2017 and 2018 in Nanning, Guangxi Province, respectively. Chr = chromosome. significantly different from the susceptible parent $(P=0.0038)$. The results were consistent with the mapping of this interval using RILs and indicate that $q S C R 6.01$ could be used to improve resistance to SCR. Five hybrid progeny of CL183 and Ye478 were selected to narrow down the target segment (Fig. 3). The target segment was finally located between markers Y6q77 and Y6q79 at a physical distance ranging from 77.6 to $79.6 \mathrm{Mb}$ (Fig. 4).

\section{Discussion}

SCR is becoming a more and more serious disease in maize, which has caused severe yield loss in tropical and subtropical maize production zones and Huang-Huai-Hai region in China in recent years. Deployment of resistant varieties is the most effective way to control this disease. Searching for the resistant maize germplasm and mining the resistance genes is the primary process used in breeding programs. QTL mapping, an efficient method for locating quantitative traits, has greatly improved breeding efficiency in maize (Chen et al. 2014). The accuracy of QTL mapping is affected by the quality of genetic maps. Thus, using high-density genetic maps in this research can improve the ability to identify recombination events and breakpoints, thereby boosting QTL mapping accuracy (Schnable et al. 2009; Varshney et al. 2009). Low-density genetic maps are inadequate for QTL mapping in current studies, by which some SCRresistant QTL on chromosomes might be lost; for example, the QTLs associated with SCR resistance on chromosomes 3, 5, 6, and 9 in Qi319 were undetected in a study by Chen et al. (2004).

In the current study, we detected five QTLs associated with SCR resistance. Two major resistance QTLs, qSCR6.01 and qSCR10.01, were stably detected in both tested environments, which had high LOD values of 26.42 and 27.93 and explained 22.98 and $24.15 \%$ of the phenotypic variation, respectively. In previous studies, the major SCR resistance genes were located on chromosome 10 . Wanlayaporn et al. (2013) detected a QTL associated with SCR resistance in RILs derived from a cross between inbred lines hA9104 and hA9035, flanked by makers umc1614 and umc1250 on bin6.04, which was

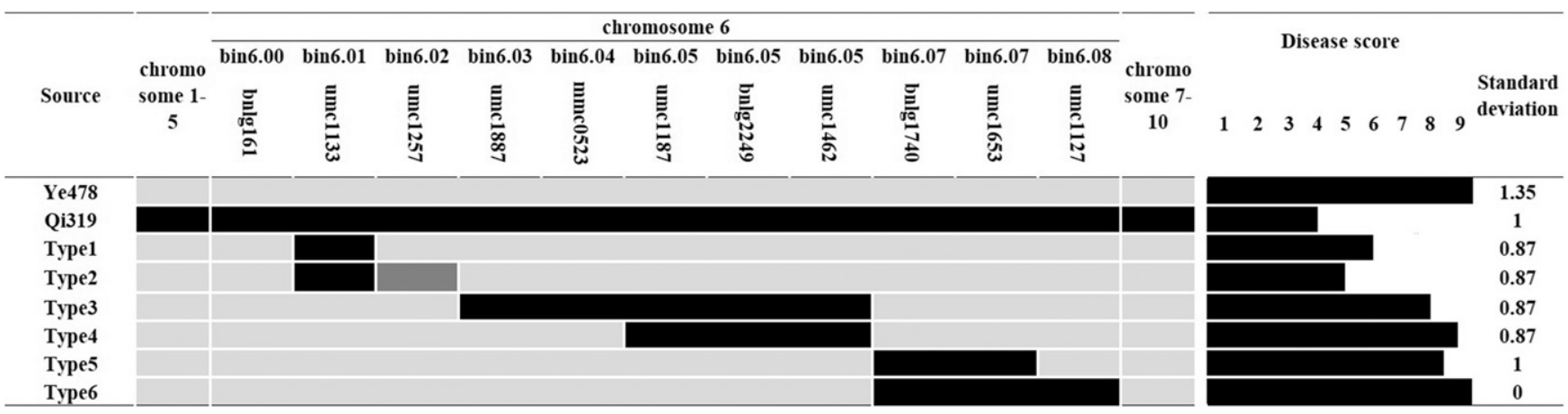

Fig. 2. Identification of $q S C R 6.01$ in a chromosome segment substitution line population derived from the cross of Qi319 and Ye478. Black and gray rectangles represent homozygous Qi319 alleles and homozygous Ye478 alleles, respectively. Light gray indicates heterozygous alleles of Qi319 and Ye478.

\begin{tabular}{|c|c|c|c|c|c|c|c|c|c|c|c|}
\hline \multirow[b]{2}{*}{ Source } & \multicolumn{10}{|c|}{ chromosome $6(70 \sim 80 \mathrm{Mb})$} & \multirow[b]{2}{*}{$\begin{array}{c}\text { Resistant } \\
\text { evaluation }\end{array}$} \\
\hline & \begin{tabular}{c}
$70.9 \mathrm{Mb}$ \\
\multirow{2}{\mathrm{a}}{} \\
ప̊
\end{tabular} & \begin{tabular}{c}
$71.4 \mathrm{Mb}$ \\
\multirow{2}{\circ}{} \\
\multirow{3}{*}{}
\end{tabular} & \begin{tabular}{c}
$73.6 \mathrm{Mb}$ \\
\multirow{2}{\mathrm{\omega}}{} \\
$\stackrel{\mathrm{\omega}}{ }$
\end{tabular} & 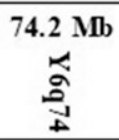 & 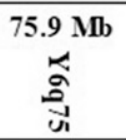 & \begin{tabular}{c}
$76.3 \mathrm{Mb}$ \\
\multirow{2}{\circ}{} \\
$\stackrel{a}{a}$
\end{tabular} & \begin{tabular}{c}
$77.6 \mathrm{Mb}$ \\
\multirow{2}{\mathrm{J}}{} \\
ป̂
\end{tabular} & 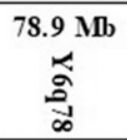 & 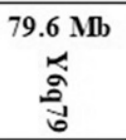 & \begin{tabular}{c}
$0.2 \mathrm{Mb}$ \\
\multirow{2}{0}{} \\
के
\end{tabular} & \\
\hline Ye478 & & & & & & & & & & & $\mathrm{s}$ \\
\hline Qi319 & & & & & & & & & & & $\mathbf{R}$ \\
\hline Type7 & & & & & & & & & & & $S, R$ \\
\hline Types & & & & & & & & & & & $\mathrm{S}, \mathrm{R}$ \\
\hline Type9 & & & & & & & & & & & $\mathrm{S}, \mathrm{R}$ \\
\hline Typel0 & & & & & & & & & & & S, R \\
\hline Typel2 & & & & & & & & & & & $\mathrm{S}$ \\
\hline
\end{tabular}

Fig. 3. Confirmation of $q S C R 6.01$ in an $F_{2}$ hybrid generation derived from the cross of CL183 and Ye478. Black and gray rectangles represent homozygous Qi319 alleles and homozygous Ye478 alleles, respectively. Light gray indicates heterozygous alleles of Qi319 and Ye478. S and R refer to susceptible and resistant, respectively. 
different from the major $q S C R 6.01$ located between markers mk2838 and mk2843 on bin6.01 in this study. Therefore, qSCR6.01 was discovered for the first time, which enriches the important genetic information for SCR resistance in maize.

Measurements of QTLs under different genetic backgrounds are not identical in various environments. These differences can be attributed to the alien chromosome segments (Eshed and Zamir 1994). The combination of RILs with CSSLs provides a powerful approach for the dissection of QTLs for SCR resistance in maize. Construction of CSSLs with the same genetic background can be used to Mendelize QTLs and, thereby, greatly improve the efficiency and possibility of individual QTL localization. To accomplish this goal, we selected Ye478 as a receptor and Qi319, which is an elite maize inbred line with resistance to diverse diseases such as southern corn leaf blight, northern corn leaf blight, SCR, and Pythium stalk rot, as a donor parent (Wang et al. 2013; Ye 2000; Zhang et al.

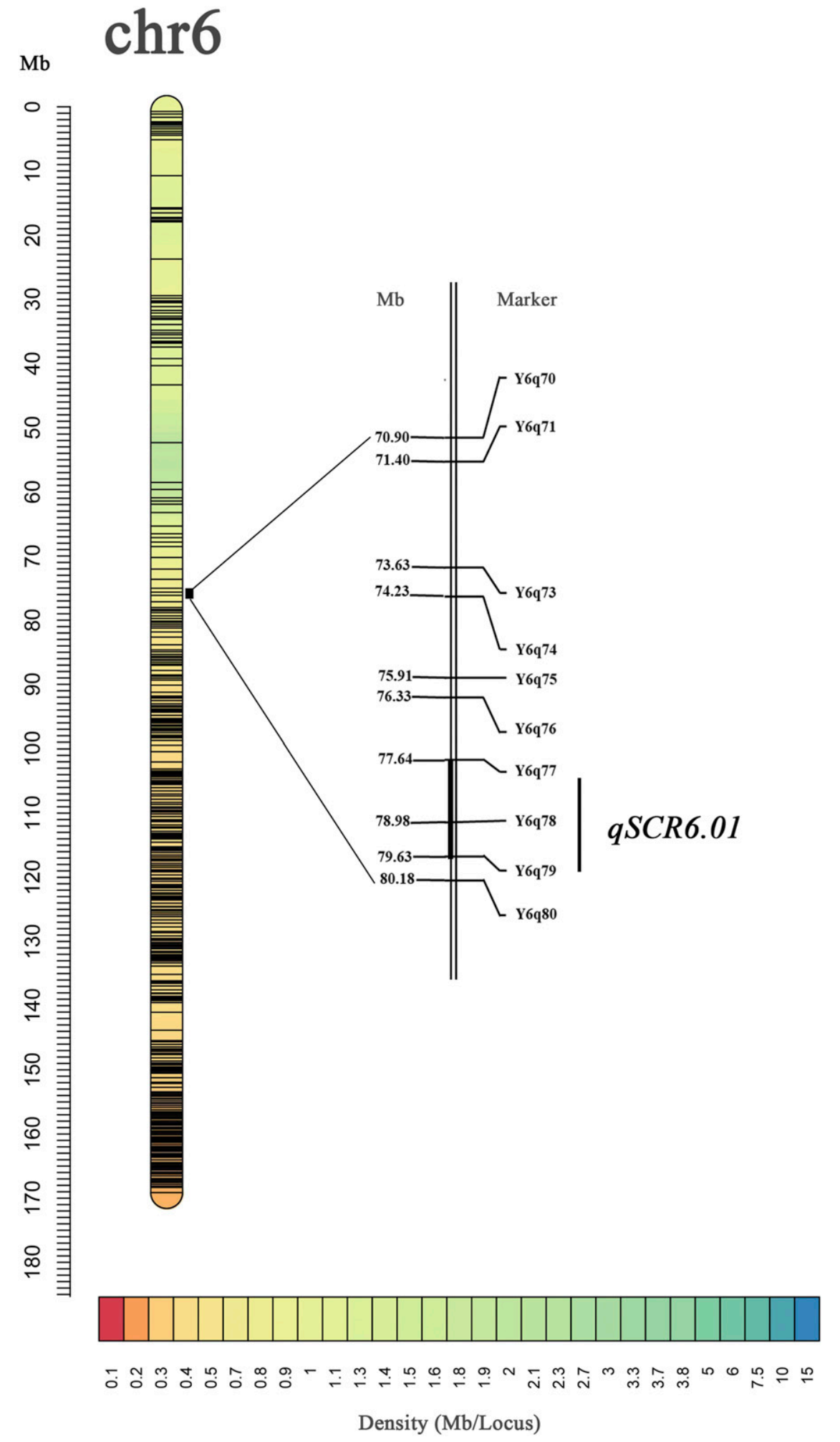

Fig. 4. Overview of locations on chromosome 6 based on the B73 RefGen_v3 sequence and increasing the marker density to 70 to 80 Mb with insertion-deletion molecular markers. 
2010). After initial positioning of QTLs in a RIL population, we carried out fine positioning using CSSLs. This approach using a RIL population and CSSLs is a new strategy for locating SCRresistance genes, and the isolated QTL can be quickly located by identifying QTL-linked markers in a CSSL population (Yung et al. 2012). CSSLs are better materials for fine mapping and assessing genetic effects (Guo et al. 2018). Because maize resistance to SCR in Qi319 is not controlled by a single gene, we used SSR markers to select six CSSLs with different genetic backgrounds on chromosome 6 but identical backgrounds on all remaining chromosomes in order to filter out the effects of other SCR-resistance genes in this study. These CSSLs were used for reconfirmation and further fine localization of the newly identified major $q$ SCR6.01. Line CL183 was found to be resistant to SCR while the other CSSLs were susceptible; therefore, CL183 can be used to construct a secondary backcross population for fine positioning and cloning of the significant gene for SCR resistance. This strategy was feasible for laying a foundation in SCR resistance breeding

Some physiological races of $P$. polysora have been reported in previous research (Hooker 1985; Ryland and Storey 1955). In China, populations of $P$. polysora exhibited relatively high genetic diversity (Guo et al. 2013). Differentiation of $P$. polysora races is difficult due to lack of differential hosts in China. In the present study, in order to keep the inoculum consistent in different environments, we selected strain PPGN collected from Nanning, Guangxi, where SCR occurs perennially, for inoculation. Of great significance for crop resistance, a variety exhibiting horizontal resistance, which is controlled by multiple genes, has the same resistance to different physiological races of a pathogen (Zhang et al. 2019). Because they express moderately durable resistance to pathogens, resistance QTLs are now often used in maize breeding for disease resistance. MAS can be effective in resistance breeding. Guodao1, an excellent disease-resistant rice variety developed by marker-assisted breeding, has been widely promoted in China (Cheng et al. 2005). In addition, the pyramiding of multiple resistance genes against powdery mildew by MAS has been found to dramatically enhance resistance to this disease (Wang et al. 2001). Pyramiding of several resistance genes in a single variety and the use of multiline cultivars are the most effective methods to improve the durability of maize SCR resistance. qSCR6.01, the resistance gene found in this study, has not been previously reported. The application of $q S C R 6.01$ in breeding can enrich genetic diversity and may reduce the incidence of disease outbreaks in large areas. Our results provide the basis for further fine mapping and, ultimately, gene cloning. Additional exploration of the molecular mechanism underlying disease resistance will be beneficial for the breeding of superior inbred lines and SCR-resistant hybrids.

\section{Acknowledgments}

We thank L. Bianji, Edanz Group China (https://www.liwenbianji.cn/ac), for editing the English text of a draft of this article.

\section{Literature Cited}

Alonso-Blanco, C., and Koornneef, M. 2000. Naturally occurring variation in Arabidopsis: An underexploited resource for plant genetics. Trends Plant Sci. 5:22-29.

Bangtai, W., Shuhong, Z., and Zhangying, X. 2012. QTL mapping for ear length based on chromosome single segment substitution lines of 87-1 zong3 in maize. J. Maize Sci. 20:9-14.

Brewbaker, J. L., Kim, S. K., So, Y. S., Manuel, L., Hyeon, G. M., Reiguang, M., Xiao, W. L., and Aleksander, D. J. 2011. General resistance in maize to southern rust (Puccinia polysora Undrew.). Crop Sci. 51:1393-1409.

Brunelli, K. R., Silva, H. P., and Aranha-Camargo, L. E. 2002. Mapeamento de genes de resistencia quantitativa a Puccinia polysora em milho. Fitopatol. Bras. 27:134-140.

Cammack, R. H. 1954. Observations in Puccinia polysora Underwood in West Africa. West Afr. Maize Rust Res. Unit Annu. Rep. 1:16-31.

Chen, C. X., Wang, Z. L., Yang, D. E., Ye, C. J., Zhao, Y. B., Jin, D. M., Weng, M. L., and Wang, B. 2004. Molecular tagging and genetic mapping of the disease resistance gene $R p p Q$ to Southern corn rust. Theor. Appl. Genet. 108: 945-950.

Chen, W. J., Li, W. C., Yang, Z. H., Sun, S. L., Wang, X. M., Zhu, Z. D. and Duan, C. X. 2018. Preliminary identification and genetic diversity analysis of maize germplasm resources for resistance to southern corn rust. J. Plant Genet. Resour. 19:225-231, 242.
Chen, W. J., Lu, L., Li, W. C., Zhang, X. J., Sun, S. L., Zhu, Z. D., Wang, X. M., and Duan, C. X. 2019. QTL Mapping for resistance to southern corn rust in maize. J. Plant Genet. Resour. 20:521-529.

Chen, Z. L., Wang, B. B., Dong, X. M., Liu, H., Ren, L. H., Chen, J., Hauck, A., Song, W. B., and Lai, J. S. 2014. An ultra-high density bin-map for rapid QTL mapping for tassel and ear architecture in a large $\mathrm{F}_{2}$ maize population. BMC Genomics 15:433.

Cheng, L. Y., Zhan, X. D., Zhuang, J. Y., and Cheng, S. H. 2005. Breeding of indica hybrid rice guodao 1 with good quality, high yield and resistance to bacterial leaf blight by marker-assisted selection technique. Hybrid Rice 20: 16-18.

Deng, C., Li, H. M., Li, Z. M., Tian, Z. Q., Chen, J. F., Chen, G. S., Zhang, X. C., Ding, J. Q., and Chang, Y. X. 2019. New QTL for resistance to Puccinia polysora Underw. in maize. J. Appl. Genet. 60:147-150.

Ding, J. Q., Wang, X. M., Chander, S., Yan, J. B., and Li, J. S. 2008. QTL mapping of resistance to Fusarium ear rot using a RIL population in maize. Mol. Breed. 22:395-403.

Doerge, R. W., and Churchill, G. A. 1996. Permutation tests for multiple loci affecting a quantitative character. Genetics 142:285-294

Duan, C. X., Jiang, K., Qin, Z. H., Sun, S. L., Song, F. J., and Wang, X. M. 2015 Identification of resistance marker genotype and genetic diversity of maize germplasm with resistance to southern corn rust. J. Plant Prot. 42:899-907.

Duan, D. R., and He, H. Z. 1984. Description of a rust Puccinia polysora on corn in Hainan Island. Acta Mycol. Sin. 2:125-126.

Elshire, R. J., Glaubitz, J. C., Sun, Q., Poland, J. A., Kawamoto, K., Buckler, E. S., and Mitchell, S. E. 2011. A robust, simple genotyping-by-sequencing (GBS) approach for high diversity species. PLoS One 6:e19379.

Eshed, Y., and Zamir, D. 1994. A genomic library of Lycopersicon pennellii in L. esculentum: A tool for fine mapping of genes. Euphytica 79:175-179.

Futrell, M. C., Hooker, A. L., and Gene, S. E. 1975. Resistance in maize to corn rust, controlled by a single dominant gene 1. Crop Sci. 15:597-599.

Guo, L. X., Shi, Y. Z., Gong, J. W., Liu, A. Y., Tan, Y. N., Gong, W. K., Li, J. W., Chen, T. T., Shang, H. H., Ge, Q., Lu, Q. W., Sun, J., and Yuan, Y. L. 2018. Genetic analysis of the fiber quality and yield traits in G. hirsutum background using chromosome segments substitution lines (CSSLs) from Gossypium barbadense. Euphytica 214:82.

Guo, Y. Y., Chen, M. G., Sun, S. L., Wu, X. F., Jing, K., Zhu, Z. D., Li, H. J., He, Y. Q., and Wang, X. M. 2013. Genetic diversity of Puccinia polysora Underw. in China. Sci. Agric. Sin. 46:4523-4533.

Holland, J. B., Uhr, D. V., Jeffers, D., and Goodman, M. M. 1998. Inheritance of resistance to southern corn rust in tropical-by-corn-belt maize populations. Theor. Appl. Genet. 96:232-241.

Hooker, A. L. 1985. Diseases, distribution, epidemiology, and control. Pages 207-236 in: Corn and Sorghum Rusts. A. P. Roelfs and W. R. Bushnell, eds. Academic Press, Orlando, FL, U.S.A.

Hospital, F., Dillmann, C., and Melchinger, A. E. 1996. A general algorithm to compute multilocus genotype frequencies under various mating systems. Bioinformatics 12:455-462.

Jines, M. P., Balint-Kurti, P., Robertson-Hoyt, L. A., Molnar, T., Holland, J. B . and Goodman, M. M. 2007. Mapping resistance to southern rust in a tropical by temperate maize recombinant inbred topcross population. Theor. Appl. Genet. 114:659-667.

Li, F., Jia, H. T., Liu, L., Zhang, C. X., Liu, Z. J., and Zhang, Z. X. 2014 Quantitative trait loci mapping for kernel row number using chromosome segment substitution lines in maize. Genet. Mol. Res. 13:1707-1716.

Li, H. H., Ye, G. Y., and Wang, J. K. 2007. A modified algorithm for the improvement of composite interval mapping. Genetics 175:361-374.

Liu, J., Ma, Q., Yu, K., and Wang, X. M. 2009. Study on the occurrence area of southern maize rust and the field resistance of maize varieties in China. Crop 3:71-75.

Liu, Y. Y., Shi, J., and Wang, Q. L. 1999. Southern corn rust occurred in Hebei Province in 1998. Plant Prot. 25:53.

Liu, Z. H., Li, H. Q., Cui, Z. T., Wu, X., Duan, L. J., Feng, X. X., and Tang, J. H 2011. QTL detected for grain-filling rate in maize using a RIL population. Mol. Breed. 27:25-36

Liu, Z. X., Wang, S. C., Dai, J. R., Huang, L. J., and Huang, H. H. 2003. Studies of genetic analysis and SSR linked marker location of gene resistance to Southern rust in inbred line P25 of maize. J. Genet. Genomics 30:706-710.

Lopez-Zuniga, L. O., Wolters, P., Davis, S., Weldekidan, T., Kolkman, J. M., Nelson, R., Hooda, K. S., Rucker, E., Thomason, W., Wisser, R., and BalintKurti, P. 2019. Using maize chromosome segment substitution line populations for the identification of loci associated with multiple disease resistance. G3 Genes Genomics Genet. 9:189-201.

Meng, L., Li, H. H., Zhang, L. Y., and Wang, J. K. 2015. QTL IciMapping: Integrated software for genetic linkage map construction and quantitative trait locus mapping in biparental populations. Crop J. 3:269-283.

Rhind, D., Waterston, J. M., and Deighton, F. C. 1952. Occurrence of Puccinia polysora Underw. in West African. Nature 169:631.

Ryland, A. K., and Storey, H. H. 1955. Physiological races of Puccinia polysora Underw. Nature 176:655-656.

Sala, R. G., Andrade, F. H., Camadro, E. L., and Cerono, J. C. 2006. Quantitative trait loci for grain moisture at harvest and field grain drying rate in maize (Zea mays L.). Theor. Appl. Genet. 112:462-471. 
Schnable, P. S., Ware, D., Fulton, R. S., Stein, J. C., Wei, F. S., Pasternak, S., Liang, C. Z., Zhang, J. W., Fulton, L., Graves, T. A., Minx, P., Reily, A. D., Courtney, L., Kruchowski, S. S., Tomlinson, C., Strong, C., Delehaunty, K., Fronick, C., Courtney, B., Rock, S. M., Belter, E., Du, F. Y., Kim, K., Abbott, R. M., Cotton, M., Levy, A., Marchetto, P., Ochoa, K., Jackson, S. M., Gillam, B., Chen, W. Z., Yan, L., and Higginbotham, J. 2009. The B73 maize genome: Complexity, diversity, and dynamics. Science 326: 1112-1115.

Storey, H. H., and Howland, A. K. 1959. Resistance in maize to the tropical American rust fungus, Puccinia polysora. Heredity 13:61-65.

Tian, Y. J., Zhao, S. G., Zhang, J., Wang, Q. Y., Huang, L. H., and Chen, H. D. 2014. Research progress on corn rust in China. Chin. Agric. Sci. Bull. 30: 226-231.

Ullstrup, A. J. 1965. Inheritance and linkage of a gene determining resistance in maize to an American race of Puccinia polysora. Phytopathology 55:425-428.

Varshney, R. K., Nayak, S. N., May, G. D., and Jackson, S. A. 2009. Nextgeneration sequencing technologies and their implications for crop genetics and breeding. Trends Biotechnol. 27:522-530.

Wang, J. J., Xu, Z. N., Yang, J., Lu, X. H., Zhou, Z. Q., Zhang, C. S., and Zheng, L. 2018. qNCLB7.02, a novel QTL for resistance to northern corn leaf blight in maize. Mol. Breed. 38:54.

Wang, J. K. 2009. Inclusive composite interval mapping of quantitative trait genes. Acta Agron. Sin. 35:239-245.

Wang, J. K. 2017. Quantitative Genetics. Science Press 3, Beijing, China.

Wang, S. L. 1999. Southern corn rust was epidemic in the provinces of Shandong, Henan and Jiangsu. Plant Prot. Technol. Dissemination 3:40-41.

Wang, X. M. 2005. Identification and investigation of resistance to pests and diseases in maize. Crops 6:53-55.

Wang, X. M., Shi, J., Jin, Q. M., Li, X. and Sun, S. X. 2010. Field Manual of Maize Diseases and Insect Pests. China Agricultural Science and Technology Press 254, Beijing, China.

Wang, X. Y., Chen, P. D., and Zhang, S. Z. 2001. Pyramiding and marker-assisted selection for powdery mildew resistance genes in common wheat. J. Genet. Genomics 28:640-646.

Wang, Y. L., Wu, J., and Wu, F. Y. 2013. Application of maize inbred line Qi319 in germplasm improvement. Bull. Agric. Sci. Technol. 2013:87-89.

Wang, Z. H., Wang, X., Zhang, L., Liu, X. J., Di, H., Li, T. F., and Jin, X. C. 2012. QTL underlying field grain drying rate after physiological maturity in maize (Zea mays L.). Euphytica 185:521-528.

Wang, Z. Q., Yu, C. Y., Liu, X., Liu, S. J., Yin, C. B., Liu, L. L., Lei, J. G., Jiang, L., Yang, C., Chen, L. M., Zhai, H. Q., and Wan, J. M. 2012. Identification of Indica rice chromosome segments for the improvement of Japonica inbreds and hybrids. Theor. Appl. Genet. 124:1351-1364.

Wanlayaporn, K., Authrapun, J., Vanavichit, A., and Tragoonrung, S. 2013. QTL mapping for partial resistance to southern corn rust using RILs of tropical sweet corn. Am. J. Plant Sci. 4:878-889.
Wisser, R. J., Balint-Kurti, P. J., and Nelson, R. J. 2006. The genetic architecture of disease resistance in maize: A synthesis of published studies. Phytopathology 96:120-129.

Wu, X. J., Li, N., Zhao, P. F., He, Y., and Wang, S. C. 2015. Geographic and genetic identification of RppS, a novel locus conferring broad resistance to Southern corn rust disease in China. Euphytica 205:17-23.

Yao, G. Q., Shan, J., Cao, B., Cui, L. G., Du, S. L., Han, Z. J., Liu, T. S., Li, C. L. and Wang, L. M. 2013. Mapping the maize southern rust resistance gene of inbred line CML470. J. Plant Genet. Resour. 14:518-522.

Ye, J. C. 2000. The first inbred lines Qi319 immune to maize southern rust bred in China. Sci. Agric. Sin. 33:110.

Young, N. D., and Tanksley, S. D. 1989. Restriction fragment length polymorphism maps and the concept of graphical genotypes. Theor. Appl. Genet. 77:95-101.

Yung, L., Ding, D., Li, W. H., Xie, H. L., Tang, J. H., and Fu, Z. Y. 2012 Construction of single segment substitution lines (SSSLs) of elite inbred lines in maize. J. Maize Sci. 20:52-55.

Zhang, C. S., Zhou, Z. Q., Yong, H. J., Zhang, X. C., Hao, Z. F., Zhang, F. J., Li, M. S., Zhang, D. G., Li, X. H., Wang, Z. H., and Weng, J. F. 2017. Analysis of the genetic architecture of maize ear and grain morphological traits by combined linkage and association mapping. Theor. Appl. Genet. 130 1011-1029.

Zhang, H., Zhao, Q., Sun, Z. Z., Zhang, C. Q., Feng, Q., Tang, S. Z., Liang, G. H., Gu, M. H., Han, B., and Liu, Q. Q. 2011. Development and high-throughput genotyping of substitution lines carrying the chromosome segments of indica 93 11 in the background of japonica Nipponbare. J. Genet. Genomics 38:603-611.

Zhang, X. L., Zhang, H. H., Xu, X. Y., Yang, H. L., Duan, Z. Y., and Yao, Z. J. 2019 Identification and analysis of leaf rust resistance genes in 12 main wheat cultivars (lines) from Hebei Province. J. Plant Genet. Resour. 20:982-990.

Zhang, Y., Xu, L., Zhang, D. F., Dai, J. R., and Wang, S. C. 2010. Mapping of Southern corn rust-resistant genes in the W2D inbred line of maize (Zea mays L.). Mol. Breed. 25:433-439.

Zheng, Y. J., Zhang, F. H., Meng, C. M., Hou, D. Y., and Sun, Z. 2009. Studies on characteristic differentiation of corn inbred line Ye478 and its related lines. Seed 28:74-75

Zhou, C. J., Chen, C. X., Cao, P. X., Wu, S. W., Sun, J. W., Jin, D. M., and Wang, B. 2007. Characterization and fine mapping of $R p p Q$, a resistance gene to southern corn rust in maize. Mol. Genet. Genomics 278:723-728.

Zhou, Z. Q., Zhang, C. S., Zhou, Y., Hao, Z. F., Wang, Z. H., Zeng, X., Di, H., Li, M. S., Zhang, D. G., Yong, H. J., Zhang, S. H., Weng, J. F., and Li, X. H. 2016 Genetic dissection of maize plant architecture with an ultra-high density bin map based on recombinant inbred lines. BMC Genomics 17:178.

Zuo, W. L., Chao, Q., Zhang, N., Ye, J. R., Tan, G. Q., Li, B. L., Xing, Y. X., Zhang, B. Q., Liu, H. J., Fengler, K. A., Zhao, J., Zhao, X. R., Chen, Y. S., Lai, J. S., Yan, J. B., and Xu, M. L. 2015. A maize wall-associated kinase confers quantitative resistance to head smut. Nat. Genet. 47:151-157. 